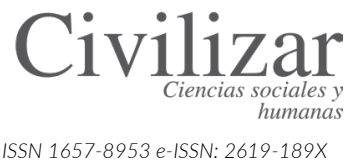

ISSN 1657-8953 e-ISSN: 2619-189X

\title{
La ciencia política en Colombia. Reflexiones sobre su quehacer investigativo*
}

\author{
The Political Science in Colombia. \\ Reflections on its research work \\ Julián Andrés Cuellar Argote \\ Sergio Huertas Hernández $z^{\ddagger}$ \\ Camila Andrea Sánchez ${ }^{\pi}$
}

* Artículo de investigación resultado del proyecto "Ciencia Política en Colombia" del grupo de Investigación YUMA-IMA Grupo Interdisciplinario de Estudios sobre el Territorio.

${ }^{\S}$ Doctor en Ciencia Política Universidad de Belgrano. Docente de Planta de la Universidad del Tolima Departamento de Ciencias Sociales y Jurídicas.

Correo electrónico:

jacuellara@ut.edu.co

(iD) 0000-0003-2257-0285

‡ Estudiante del Doctorado en Ciencia Política Pontificia Universidad Católica

de Chile. Maestría en Política Comparada FLACSO Ecuador. Politólogo Universidad del Tolima.

Correo electrónico:

sahuertas26@gmail.com

(iD) $0000-0002-2697-3068$

" Estudiante del Doctorado en Ciencias Sociales FLACSO México. Maestría en Sociología Universidad Iberoamericana Ciudad de México. Docente de Zion internacional University y academia internacional de formación en ciencias forenses. Correo electrónico:

casanchezs1018@gmail.com

(iD) $0000-0002-4152-8675$

Cómo citar:

Cuéllar-Argote, J., Huertas, S., y Sánchez,

C. (2020). La Ciencia Política en Colombia. Re lexiones sobre su quehacer investigativo. Civilizar: Ciencias Sociales y Humanas, 2o(39), 69-84. https://doi. org/10.22518/jour.ccsh/2020.2a04

Recibido: 10/01/2020

\section{Resumen}

El artículo se centra en analizar el estado de la investigación científica de la ciencia política en Colombia. Por lo tanto, la investigación ahonda en el examen de los grupos que se encuentran reconocidos y avalados por Colciencias. De esta manera, se provee de información útil sobre las áreas de estudio en las que se encuentran enfocados los grupos de investigación en el país, así como la ubicación geográfica, el carácter público o privado y el género de los miembros adscritos. Entre los principales hallazgos se da cuenta de una disciplina orientada principalmente en la investigación en conflicto, seguridad y paz, junto a relaciones internacionales. A su vez, se evidencia una desatención en los temas de política comparada, metodología y economía política. Finalmente, el estudio plantea la necesidad de propender un mayor liderazgo de mujeres frente a los grupos y consolidar la investigación en ciencia política fuera de las principales urbes de Colombia.

\section{Palabras clave}

Colombia, ciencia política, grupos de investigación, áreas de estudio, género.

\section{Abstract}

The article focuses on analyzing the state of scientific research in political science in Colombia. Therefore, the investigation delves into the examination of the groups that are recognized and endorsed by Colciencias. In this way, useful information is provided on the areas of study in which the research groups in the country are focused, their geographical location, the public or private character and the gender of the members assigned to them. Among the main findings, we discovered a discipline focused mainly on conflict, security and peace research, together with international relations. At the same time, there is a lack of attention in the topics of comparative politics, methodology and political economy. Finally, the study raises the need to promote greater leadership of women in front of the groups and consolidate research in political science outside the main cities of Colombia.

\section{Keywords}

Colombia, political science, research groups, areas of study, gender. 


\section{Introducción}

Hablar de la ciencia política como disciplina remite a la revisión de los acuerdos realizados en el período de la posguerra por la Organización de las Naciones Unidas para la Educación, la Ciencia y la Cultura (UNESCO). En aquella ocasión fueron designadas las ciencias sociales como las disciplinas encargadas de brindar explicaciones a los sucesos cotidianos y desarrollar reflexiones ante cualquier tipo de acontecimiento que atentara contra la seguridad y la paz mundial. Fue así que, a partir del año 1948, se fueron sentando las bases para el posterior surgimiento de programas de estudio universitario en ciencia política alrededor del mundo. Junto a ello, se evidenció una intensificación en la producción de libros y artículos que abordaban la discusión sobre el estudio de la política como disciplina científica, sus acuerdos, campo de estudio, áreas, gremios y asociaciones (Bulcourf y Vázquez, 2004) ${ }^{\mathbf{1}}$.

Así, la ciencia política empieza a abrirse camino en las disciplinas científicas del mundo y con el pasar de los años se consolida como una ciencia con capacidad y bagaje explicativo de la realidad política, sin que ello implicara el abandono a la reflexión sobre su quehacer, sus métodos y su campo de estudio (Bejarano y Wills, 2005; Cuellar Argote, 2007; Cuellar Argote y Caicedo Ortiz, 2015; Duque Daza, 2015). Dichas discusiones se mantienen vigentes puesto que las ciencias sociales no son estáticas, sino que obedecen a los cambios constantes de las realidades y los contextos (Caicedo Ortiz et al., 2015). En este sentido, teniendo en cuenta el permanente proceso de redefinición en las ciencias sociales -el cual no resulta ser un asunto lineal-, vale la pena preguntarnos por el estado de la investigación de la ciencia política colombiana, dado que es uno de los aspectos que marca la pauta a la hora de hablar de la institucionalización de una disciplina ${ }^{2}$. A su vez, resulta que dicho examen ofrece elementos dilucidadores de los temas que investigan los politólogos del país, que contribuirán al debate generado en el Manifiesto de Popayán (2017) sobre la investigación y la divulgación dentro del campo de la ciencia política en América Latina.

La pregunta por responder en el presente artículo es, entonces, ¿cuáles son las principales áreas de estudio declaradas por los grupos de investigación en ciencia política en Colombia? De esta pregunta se desprenden algunos interrogantes adicionales que atañen al quehacer investigativo del politólogo colombiano, como lo son ¿cuál es el carácter institucional de las universidades donde se encuentran los grupos?, ¿cómo se distribuyen geográficamente estos grupos? y ¿qué tanta paridad de género existe en los grupos de investigación?

El análisis que desarrollamos en las siguientes líneas resulta de interés para la comunidad académica puesto que, de manera general, el estudio sobre el estado de la disciplina en Colombia se ha concentrado en examinar las áreas de los planes de estudio, la oferta de programas que se ofrecen en el país o la producción de conocimiento en revistas especializadas (Duque Daza, 2014; Losada, 2004). Por lo tanto, la pesquisa al plantear un análisis sobre los grupos de investigación provee retos y desafíos sobre uno de los tres frentes de institucionalización -formación, profesionalización e investigaciónde la ciencia política que atañe precisamente a la investigación (Altman, 2005; Angel Baquero et al., $2019)^{3}$. De este modo, encontramos que es necesario dar cuenta de las áreas de estudio de los grupos de investigación con el fin de motivar el examen sobre los mismos en otros países de la región (Barrientos del Monte, 2015; Leiras et al., 2005).

Con el objetivo de dar respuesta a los cuestionamientos que hemos planteado, el artículo, luego de la introducción, dialoga sobre cuáles son las áreas de estudio de la ciencia política como disciplina. En la segunda sección, se presenta el estado de las áreas que desarrollan los grupos de investigación de ciencia política en el país, aclarando que solamente se toma lo referido a los objetivos y las líneas de trabajo inscritas por los grupos que se encuentran registrados y avalados por Colciencias ${ }^{4}$. En la tercera sección se explora la manera como están ubicados geográficamente estos grupos de investigación, así como el carácter de las universidades (públicas-privadas) en donde se encuentran y la relación de género inmersa en dichos grupos. Finalmente, en la última sección planteamos las conclusiones obtenidas con el estudio junto a ciertos lineamientos que se deben suscitar entorno a la investigación de la ciencia política colombiana.

\section{Áreas de estudio de la ciencia política}

En el desarrollo de la ciencia política como disciplina de formación e investigación no existe como tal un consenso generalizado sobre un número total de áreas de conocimiento o formación del politólogo (Cuellar Argote y Díaz Martínez, 2019). No obstante, alrededor del siglo XX se han desarrollado unos 
acuerdos mínimos que se reflejan en los currículos de las diversas universidades que otorgan el título de politólogo (Laitin, 2004; Bentancur y Mancebo, 2017). Por lo tanto, en el presente apartado se exponen las diferentes propuestas sobre las áreas de especialización de los politólogos. Así, para examinar las áreas de estudio de la disciplina, dividimos la discusión del acápite en lo que hemos llamado los tres momentos de las áreas. La importancia de evidenciar las áreas es que, a partir de su consolidación, se va configurando y replanteando la ciencia política como disciplina y se van integrando en su quehacer investigativo, profesional y metodológico. Realizada esta observación, a continuación, describimos cada uno de los tres momentos que nos permiten conocer cuáles son las áreas que conforman la ciencia política como disciplina académica.

\section{Primer momento}

En este primer momento se encuentran los acuerdos concitados a partir de la denominada "lista tipo" promovida después de la segunda guerra mundial por petición de la UNESCO. Dicha lista se elabora gracias al esfuerzo de expertos y estudiosos sobre el estatuto científico, epistemológico y ontológico de la ciencia política. La publicación de ella se llevó a cabo luego de reuniones de expertos y cultores de las ciencias sociales realizadas en Paris sobre el año de 1948 (Bulcourf y Vázquez, 2004). La lista tipo estaba compuesta por cuatro secciones -teoría política, instituciones políticas, partidos políticos y relaciones internacionales- que definían a la ciencia política como disciplina académica. Dichas áreas marcaron el punto de referencia a la hora de ofertar programas universitarios y, por ende, elaborar currículos en ciencia política (Prelot, 1964). A partir de la lista tipo se encuentran las áreas de la ciencia política que se pueden considerar como aquellas estrictamente disciplinares y que hacen alusión al objeto de estudio de la ciencia política. En ese primer momento fueron reunidos los autores que proponían áreas de conocimiento de la disciplina a partir del acuerdo generado por la UNESCO, añadiendo algunas modificaciones mínimas referentes a su nomenclatura.

Dentro del compendio de académicos que realizaron estudios sobre las áreas de la ciencia política a partir de los lineamientos desarrollados en la lista tipo, se encuentran trabajos en los que, a su vez, se plantea ampliar el espectro de las áreas. Una evidencia de ello es el trabajo desarrollado por Greens- tein y Polsby (1975), el Handbook of Political Science, en el cual los autores realizaron un replanteamiento de las cuatro secciones reconocidas por la UNESCO. Los autores ya no las pensaban como secciones, sino como campos de fragmentación de la disciplina, sugiriendo con ello, ya no cuatro sino siete áreas que componían la ciencia política. Por otra parte, Harto de Vera (2006) propuso pensar la ciencia política en torno a cinco áreas de estudio. Este mismo autor señala la importancia del trabajo realizado por parte de la UNESCO, puesto que - gracias a la lista tipose crea la Political Studies Association que permite reconocer una disciplina ante la sociedad ${ }^{5}$.

Sin embargo, es oportuno señalar que en este primer momento se evidencia principalmente una ciencia política pensada desde una mirada de índole institucional y gubernamental. Por lo tanto, al analizar lo propuesto por los autores se reconoce que existen vacíos al momento de proponer áreas referidas a temas metodológicos y de procesos colectivos. Tal situación se puede explicar por el contexto de conmoción social y político vivido en las guerras mundiales, en donde el llamado de atención se enfatizaba especialmente en los procesos políticos por desarrollarse desdelas instituciones. Esteénfasis tenía como fin teorizar y brindar explicaciones a los acontecimientos antidemocráticos que habían germinado en la primera mitad del siglo veinte (Prelot, 1964). En tal sentido, lo que se deseaba con las propuestas desarrolladas para este primer aspecto era propiciar un conjunto de lineamientos desde los cuales se generaran argumentos para no repetir dichos procesos conflictivos entre los países.

\section{Segundo momento}

Pasamos entonces de lo institucional, como base para comprender los inicios de la disciplina, a plantear las áreas de la ciencia política en términos de formación y profesionalización del politólogo. En este segundo momento se presenta una reflexión a lo que es la interdisciplinariedad, configurada y estructurada en la segunda mitad del siglo veinte e inicios del veintiuno (Bentancur y Mancebo, 2017). Al respecto, cabe mencionar que es en este momento en el que se encuentra el mayor compendio de trabajos que investigan sobre las áreas de la disciplina. Esta tendencia obedecía a la presencia de una mayor masa crítica y constructiva de académicos preocupados por el devenir de una disciplina que se encontraba en un estado de consolidación (Leyva y Ramírez, 2015). 
En esta sección, se destacan trabajos como el de Goodin y Klingemann (1996), A New Handbook of Political Science, quienes retomaron la discusión sobre las áreas de estudio de la disciplina. Los autores argumentan que la clasificación de estas áreas obedece a los procesos de profesionalización y especialización de los politólogos, y propusieron una nueva clasificación con ocho subdisciplinas, las cuales se desprenden de una gran disciplina llamada ciencia política. Un punto importante en este trabajo es que, por primera vez, se reconoció a la política comparada como área de estudio de la ciencia política. Es precisamente a partir de este trabajo que otros autores empiezan a reconsiderar la política comparada como parte fundamental para la formación y profesionalización del politólogo.

Posteriormente, aparece Dieter Nohlen (2003) con su texto Cómo enseñar ciencia política, el cual, entre otras cosas, se enfocó en el análisis de la enseñanza de la disciplina. Para responder a las diversas preguntas que guían su texto, Nohlen realizó una revisión sobre las áreas o cuerpo de conocimiento de la ciencia política. En tal sentido, este autor formuló tres áreas que integran el estudio de la disciplina: la teoría política, las relaciones internacionales y la política comparada. De acuerdo con Nohlen (2003), esta es una terna que promueve un análisis de los procesos políticos e internacionales desde reflexiones teóricas. Además, remarcó la idea de que la ciencia política es relacional y debe integrar dimensiones, enfoques y métodos adquiridos con las relaciones internacionales.

Por otra parte, David Laitin (2004) emprendió un trabajo sobre las principales universidades que estuviesen enseñando ciencia política en Estados Unidos, titulado The Political Science Discipline. Este análisis comprende un estudio aleatorio al plan de estudios de treinta y cuatro instituciones que enseñaban ciencia política a nivel de pregrado. A partir de ello, Laitin afirmó que existe una anarquía $\mathrm{y}$ un caos disciplinar que se sustentan en la muestra analizada y en la multiplicidad de mesas temáticas que se han venido propagando en los congresos organizados por la Asociación Internacional de Ciencia Política (IPSA). El autor sugirió que podemos ordenar académicamente este "caos disciplinar" y agrupar las diversas líneas en seis subcampos; sin embargo, se dejan a un lado ciertos aspectos como lo son los partidos políticos, los procesos colectivos y la reflexión filosófica de la política.
En el 2006, Josep Vallés, en su libro Ciencia política: una introducción, realizó un trabajo sobre los campos temáticos propios de la ciencia política. El autor mencionó que, al igual que en las ciencias naturales y las ciencias sociales, en la ciencia política se ha dado lugar a un proceso de ramificación que ha hecho más frondoso el clásico árbol de la disciplina. De acuerdo con Vallès (2006, p. 65), los diferentes campos temáticos han permitido el surgimiento de una serie de subdisciplinas que se han venido desarrollando en los centros de investigación, en los departamentos docentes y en los planes de estudio de las universidades. Realizada esta precisión, el autor propuso una serie de campos temáticos que son propicios para la formación de los politólogos (ver tabla 1).

En el caso colombiano, uno de los pioneros en poner sobre la mesa el tema de las áreas es Cuellar Argote (2007). El autor, en su libro Un diagnóstico a la enseñanza de la Ciencia Política en Colombia, evidenció que en la formación de los politólogos colombianos predominan algunas materias como lo son historia, economía, derecho, relaciones internacionales, teoría de la administración y filosofía. Es decir, aquellas con las que existe mayor cercanía o, en el mejor de los casos, con las que se encuentran afinidades. De este examen realizado a los programas de pregrado, se desprendió una propuesta sobre las áreas que obedecen a la formación de los politólogos del país. A este listado de áreas, Cuellar Argote lo denominó disciplinas, las cuales se agrupan alrededor de unas áreas de formación impartidas por los programas de pregrado universitarios. El resultado fue un listado de diecisiete áreas.

En el 2010, Goodin realizó una reformulación de las subdisciplinas propuestas por él junto a su colega Klingemann en 1975. En este trabajo, Goodin (2010) realizó ciertas modificaciones que obedecieron a los campos de profesionalización y especialización de los politólogos. Como resultado, se ve la necesidad de agregar nuevas áreas que se incorporan siguiendo los procesos actuales que afrontan los politólogos de la época. En esta misma lógica de reconstrucción de las áreas, Buitrago et al. (2012) reelaborarían el trabajo desarrollado por Vallès (2006) anteriormente descrito. Este trabajo consistió en agrupar algunos de los campos propuestos por Vallès (2006) para presentar lo que -a consideración de los autores- serían los campos temáticos 
de la ciencia política de una manera más lacónica. Claro está que Buitrago et al. (2012) se animarían por poner en consideración ciertas áreas que no fueron propuestas en el trabajo de Vallès (2006).

Posteriormente, Leyva (2013) realizaría un trabajo en el que, mediante un análisis empírico al currículo de los programas de pregrado en Ciencia Política de Colombia, se someten a comparación algunos de los trabajos de los autores anteriormente descritos. Cabe mencionar que este estudio es muy similar al formulado por Cuellar Argote (2007), tan solo que el autor propuso una taxonomía compuesta por 10 áreas de formación de los politólogos. Con lo mencionado hasta el momento, se puede observar que cada uno de los autores expuestos en este segundo momento de las áreas presentaron sus propuestas como el deber ser de la formación del politólogo. Además, los autores crearon estas taxonomías pensando en dar cierto orden y criterios de clasificación a los currículos impartidos por los programas de pregrado en ciencia política.

\section{Tercer momento}

El tercer momento de las áreas se establece a partir de las agendas investigativas de la disciplina y la producción académica. Por ello, en este aspecto se piensan las áreas de la ciencia política en términos de acuerdos y arreglos de las asociaciones de politólogos, quienes sintetizan estas áreas en pro del quehacer investigativo y la producción académica intelectual. Esta síntesis se obtiene como el resultado de agendas de investigación globales, regionales y nacionales, presentadas y discutidas en congresos y eventos académicos. A su vez, en este tercer aspecto se traen a colación aquellos trabajos en los que se exponen las áreas de la ciencia política en pro de impeler exámenes y análisis a la producción intelectual formulada en libros y revistas especializadas en la disciplina. Junto a lo anterior, también resulta necesario reconocer que,

una disciplina puede identificarse, entre otras cosas, por la apropiación y transmisión de un determinado saber, el cual denominaremos área temática. Cada una de ellas, aclama y defiende los límites de estas áreas temáticas, sin embargo, como es el caso que nos corresponde, las fronteras entre dichas áreas temáticas son difusas y dan pie a la unión de lo que en apariencia son dos o más disciplinas en una sola. (Cuellar Argote y Diaz Martínez, 2019, p. 19)

Desde esta perspectiva, resulta claro significar en primera medida las áreas sobre las cuales la
International Political Science Association (IPSA) llama a sus congresos mundiales. Al revisar el portal web de la IPSA, se evidencia que la Asociación organiza los paneles y sesiones de acuerdo con sus comités de investigación, los cuales -al momento de escribir este trabajo- ascienden a más de $50^{6}$. Desde una perspectiva más regional, la Asociación Latinoamericana de Ciencia Política (ALACIP) desarrolla sus congresos a partir de las líneas que trabajan sus grupos de investigación, que a la fecha llegan a sumar más de 20, tal como se puede evidenciar en la página web de ALACIP ${ }^{7}$.

Teniendo en cuenta las consideraciones de las áreas que elaboran las asociaciones para sus congresos, se hace pertinente mostrar en este tercer aspecto los trabajos sobre la producción académica de artículos en revistas indexadas y libros en la disciplina. De esta manera, se hallan trabajos como el de Leyva y Ramírez (2015), en el que se encuentra consignada una revisión empírica del conocimiento producido por la disciplina. Los autores llevarían a cabo la construcción de una base de datos de publicaciones que incluye 730 artículos de revistas y 239 libros en el período 2005-2014. Cabe anotar que, para la organización de la base de datos, fue necesario crear un listado de áreas de la ciencia política a partir de unos criterios esbozados por los autores. En tal sentido, se obtuvo un listado de 13 subdisciplinas, bajo las cuales se agrupan los libros y artículos de revistas de la muestra.

Desde esta perspectiva, Duque Daza (2015) desarrolló un trabajo en el que enumeró la producción académica de artículos científicos en doce revistas colombianas con publicación periódica en la disciplina. Se examinaron un total de 1833 artículos, para los cuales el autor realizó una división propia de doce subcampos disciplinares. Con ello se observó en qué campos se observaba una mayor producción académica y en qué campos, débil producción intelectual (Duque Daza, 2015). En esta misma línea, enfocada ya de manera regional Basabe-Serrano y Huertas-Hernández (2018) plantearon un análisis a las revistas incluidas en los índices de Scimago que versan sobre la disciplina. Gracias a este trabajo, se dio cuenta de una clasificación de 23 áreas, las cuales -a partir del examen a 531 artículos- revelaron que hay pocos estudios desarrollados en el área de metodología.

Considerando los tres aspectos abordados y las diferentes áreas expuestas en cada uno, a continua- 
ción, la tabla 1 presenta el compendio de áreas que, como se discutió anteriormente, se centran en la enseñanza de la ciencia política. De esta manera, se hace referencia a la recurrencia de esas áreas en los diferentes trabajos a los que hemos aludido hasta el momento.

Tabla 1

Recurrencia de las áreas a partir de los tres aspectos de análisis

\begin{tabular}{|c|c|c|c|c|c|c|c|c|c|c|c|c|c|c|}
\hline Áreas & 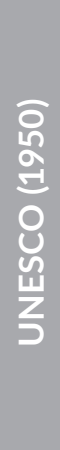 & 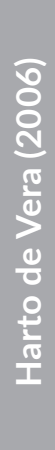 & 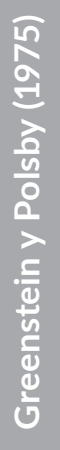 &  & 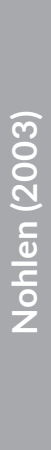 & 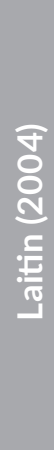 &  & 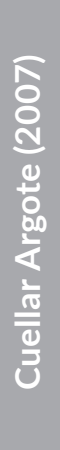 & $\begin{array}{l}\text { 을 } \\
\text { 웡 } \\
\text { 등 } \\
\stackrel{0}{0}\end{array}$ & 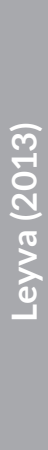 & 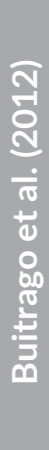 & 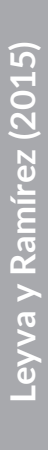 & 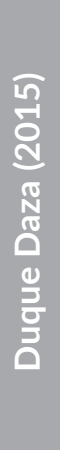 & 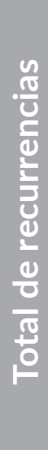 \\
\hline Teoría política & $x$ & $x$ & $x$ & $x$ & $x$ & $x$ & $x$ & $x$ & $x$ & $x$ & $x$ & $x$ & $x$ & 15 \\
\hline $\begin{array}{l}\text { Instituciones } \\
\text { políticas }\end{array}$ & $x$ & $x$ & $x$ & $x$ & & $x$ & $x$ & & $x$ & & & $x$ & $x$ & 11 \\
\hline $\begin{array}{c}\text { Partidos } \\
\text { políticos y } \\
\text { comportamiento } \\
\text { electoral }\end{array}$ & $X$ & & & $x$ & & & $x$ & $x$ & $x$ & $x$ & $x$ & $x$ & $x$ & 11 \\
\hline $\begin{array}{l}\text { Movimientos } \\
\text { sociales y acción } \\
\text { colectiva }\end{array}$ & & & & & & & $x$ & $x$ & & $x$ & $x$ & $x$ & $x$ & 8 \\
\hline $\begin{array}{c}\text { Relaciones } \\
\text { internacionales }\end{array}$ & $X$ & & $x$ & $x$ & $x$ & $x$ & $x$ & $x$ & $x$ & $x$ & $x$ & $x$ & & 13 \\
\hline $\begin{array}{l}\text { Derecho político } \\
\text { / Política judicial }\end{array}$ & & $x$ & & & & & & $x$ & $x$ & $x$ & $x$ & & & 7 \\
\hline $\begin{array}{l}\text { Metodología de } \\
\text { la ciencia política }\end{array}$ & & & $x$ & $x$ & & $x$ & & $x$ & $x$ & & & $x$ & & 8 \\
\hline $\begin{array}{l}\text { Gobierno, } \\
\text { administración } \\
\text { y políticas } \\
\text { públicas }\end{array}$ & & $x$ & $x$ & $X$ & & $x$ & $x$ & $x$ & $x$ & $x$ & & $x$ & $x$ & 12 \\
\hline $\begin{array}{c}\text { Política } \\
\text { comparada }\end{array}$ & & & & $x$ & $x$ & $x$ & $x$ & & $x$ & & & $x$ & & 8 \\
\hline $\begin{array}{l}\text { Economía } \\
\text { política }\end{array}$ & & & & $x$ & & & & $X$ & $x$ & $x$ & & & & 5 \\
\hline $\begin{array}{c}\text { Conflicto, } \\
\text { seguridad y } \\
\text { construcción de } \\
\text { paz }\end{array}$ & & & & & & & & & & & & $x$ & $x$ & 4 \\
\hline Filosofía política & & & & & & & $x$ & $x$ & & & $x$ & & & 4 \\
\hline
\end{tabular}

Fuente: elaboración propia con base en Unesco (1950), Greenstein y Polsby (1975), Harto de Vera (2006), Goodin y Klingemann (1996), Nohlen (2003), Laitin (2004), Vallès (2006), Cuellar Argote (2007), Goodin (2010), Buitrago et al. (2012), Leyva (2013), Leyva y Ramírez (2015) y Duque Daza (2015). 
En suma, con lo esbozado hasta el momento, se desea significar el proceso por el cual la disciplina tuvo que afrontar para lograr la sustentación y validación de estas áreas que la conforman. Teniendo en cuenta estas claridades, se da paso al siguiente apartado en el que se muestra, a la luz de las áreas, cómo están conformados los grupos de investigación en ciencia política en Colombia. Esta exposición se realiza como un paso en búsqueda de la comprensión de aspectos de estudio teóricos que permitan generar discusiones profundas y reflexivas de acuerdo con lo esbozado en el Manifiesto de Popayán (2017).

\section{Organización de los grupos de investigación en ciencia política en Colombia de acuerdo con las áreas de estudio de la disciplina}

Para elaborar una descripción sistemática sobre lo que se investiga en ciencia política en Colombia, se tomó como muestra los 66 grupos de investigación registrados en GrupLAC a la fecha de escribir el presente artículo. Con el fin de procurar dar respuesta a la pregunta ¿cuáles son las principales áreas de estudio declaradas por los grupos de investigación en ciencia política en Colombia? y para la selección de la muestra, se utilizó la siguiente metodología. En primera medida, accedimos al enlace proporcionado por Colciencias denominado Buscador de Colciencias, ruta Grupos. En el buscador introdujimos el sintagma ciencia política. De los 4104 resultados obtenidos, filtramos por área y seleccionamos el área Ciencias Políticas. Con ello, el resultado se redujo a los 66 grupos de investigación en ciencia política que conforman nuestra muestra. Acto seguido, analizamos cada grupo, cotejando los objetivos y las líneas de investigación por ellos declaradas. De este trabajo, se analizó la afinidad de las áreas que los grupos de investigación declararon objetivo con las áreas de la tabla 1. La figura 1 expone los resultados de este proceso. Si bien esta metodología es netamente descriptiva, es útil para dar a conocer de manera sistemática qué se está investigando en la ciencia política colombiana y cuáles son las áreas principales que se observan -tanto en la disciplina de manera general, como en los diferentes grupos de investigación registrados ante Colciencias-.

Figura 1

Cotejo entre el objetivo de los grupos de investigación y las áreas de estudio de la Ciencia Política

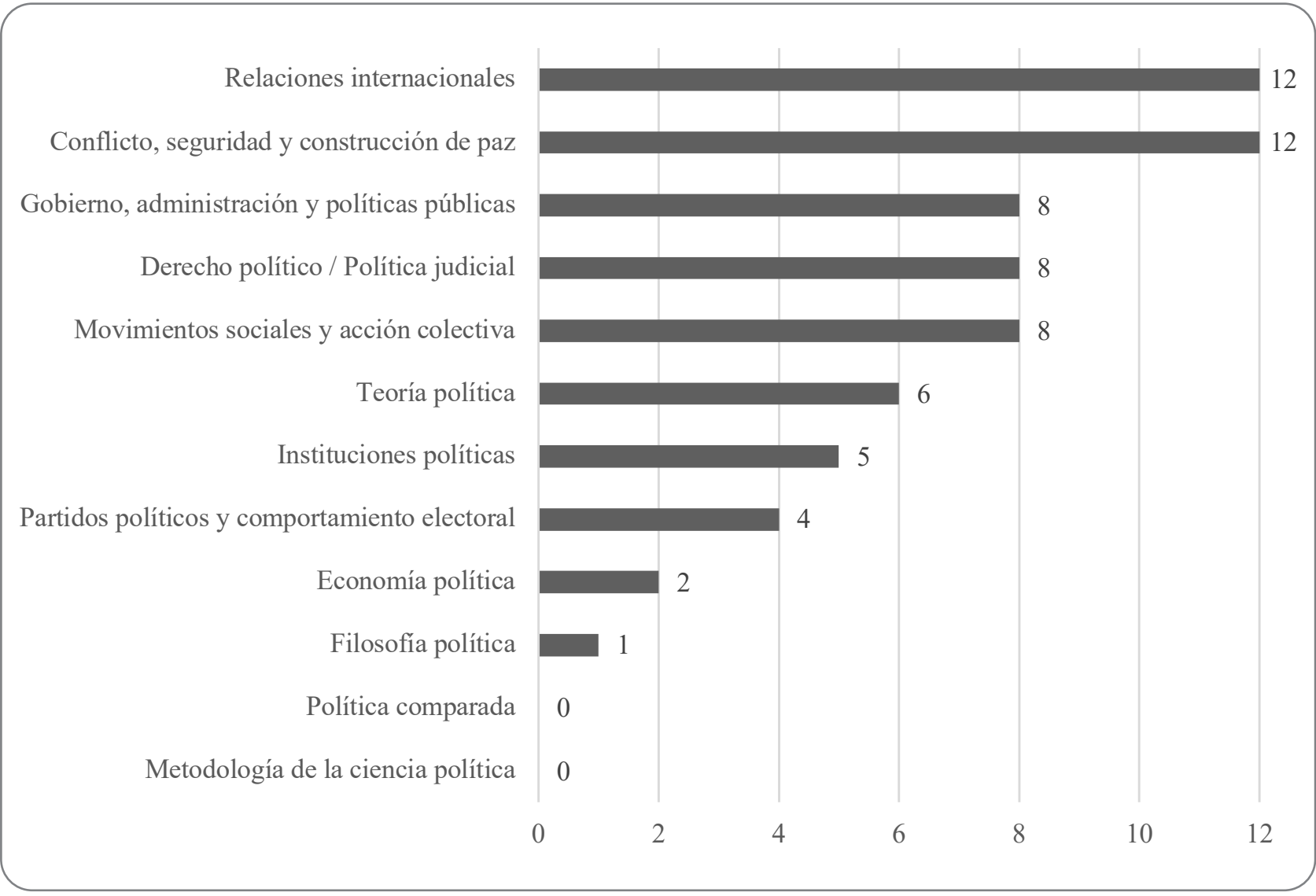

Fuente: elaboración propia con base en los resultados obtenidos por el Buscador Colciencias (2019). 
La revisión de los grupos de investigación es importante puesto que a partir de ella logramos obtener los datos que se presentan en la figura 1. En esta figura se evidencia que 24 de los 66 grupos de investigación en Colombia centran sus pesquisas principalmente en las áreas de relaciones internacionales, conflicto, seguridad, construcción de paz y posconflicto. Tal situación era de esperarse dado que la ciencia política, como lo menciona Ravecca (2019), es una ciencia que obedece en sus análisis a los contextos y realidades políticas de cada país. En tal sentido, teniendo en cuenta las diversas problemáticas del conflicto, la lucha contra el narcotráfico y la construcción de paz, no sorprende que los politólogos colombianos se vean ampliamente influidos en realizar estudios desde estas dos áreas.

La importancia evidente del área de las relaciones internacionales dentro de los grupos de investigación obedece a la diversidad de nominaciones en los pregrados de ciencia política. En los programas de pregrado se observa una cantidad considerable de etiquetas como 'ciencia política' o 'estudios políticos', 'ciencia política' y/o 'gobierno', 'ciencia política' y 'relaciones internacionales'. La investigación de Duque Daza en 2014 para Colombia hizo mención a 31 pregrados en el campo de la ciencia política; 21 de ellos con denominación en ciencia política y estudios políticos; 5 con el complemento en relaciones internacionales y 6 en Gobierno. La cantidad de grupos de investigación especializados en relaciones internacionales obedece a que las universidades en Colombia manejan lo conocido como énfasis o áreas de profundización. En estas áreas se encuentran, a su vez, gestión pública/gobierno/políticas públicas, relaciones internacionales, cultura de paz/conflicto y comunicación política/marketing político.

Es así como, gracias al estudio de Duque Daza (2014), se reconoce que el $40 \%$ de los programas de pregrado en Colombia adopta el énfasis en relaciones internacionales, de los cuales 8 tienen grupos en esa área registrados ante Colciencias. Con ello, encontramos una relación entre nominación de programas, énfasis y existencia de grupos de investigación. Otro argumento un poco más práctico para reconocer la relevancia del área de las relaciones internacionales en los grupos registrados es que, desde el inicio de la ciencia política, y según los datos de la tabla 1, hay un consenso por considerarla una de las áreas principales de la disciplina. Este acuerdo tácito se debe al carácter interdisciplinar y al trabajo cooperativo que la ciencia política realiza en el análisis del comercio exterior, las relaciones entre países, los juegos de poder entre organismos y países, entre otros.

Se evidencia también una fuerte ubicación de grupos en las áreas de gobierno, administración y políticas públicas, política judicial, movimientos sociales y acción colectiva. La explicación al hecho de que 24 grupos configuren sus objetivos de investigación en estas áreas obedece, en cierta medida, a la gran influencia que sociólogos, administradores públicos y abogados tuvieron sobre la ciencia política, por cuanto estos profesionales eran quienes la enseñaban. Tal como lo señalan Bejarano y Wills (2005), la ciencia política colombiana se fundamentó en la vocación docente de aquellos profesionales, pues formaron a las primeras generaciones de politólogos de universidades como la Universidad de Los Andes, la Universidad Javeriana, la Universidad del Cauca y la Universidad Nacional de Colombia. Es gracias a la influencia de los primeros docentes universitarios en ciencia política, que los politólogos que realizaron sus maestrías y doctorados tanto dentro como fuera del país desarrollaran sus investigaciones desde estas áreas.

Con una menor recurrencia se encuentran los grupos que focalizan sus objetivos en las áreas de economía política y filosofía política, y se encuentra un vacío investigativo en las áreas de metodología de la ciencia política y política comparada. Estos resultados, para el caso colombiano, no resultan ajenos a la realidad investigativa de América Latina. Gracias al trabajo bibliométrico realizado por Basabe-Serrano y Huertas-Hernández (2018), es posible confirmar que es precisamente en estas áreas en las que hay una menor producción de artículos académicos publicados en revistas latinoamericanas de alto impacto. En esta misma línea, algo que llama la atención es que no hay grupos registrados que tengan por objetivo y como línea de investigación ahondar sobre estos temas. Por lo tanto, cabe hacer un llamado a subsanar este vacío desde las universidades; se deben impartir cátedras al respecto e incentivar la creación de productos académicos y grupos que promuevan la discusión sobre estas temáticas.

Para observar con un poco más de claridad la relación existente entre las líneas de investigación 
declaradas y el nombre de los grupos de investigación frente a las áreas, se presenta la figura 2. Esta figura también permite observar con más detalle las áreas en las que los grupos de investigación presentan un mayor interés a la hora de realizar sus respectivas investigaciones.

\section{Figura 2}

Áreas de la ciencia política confrontadas con los objetivos de los grupos de investigación y las líneas de investigación declaradas por estos grupos

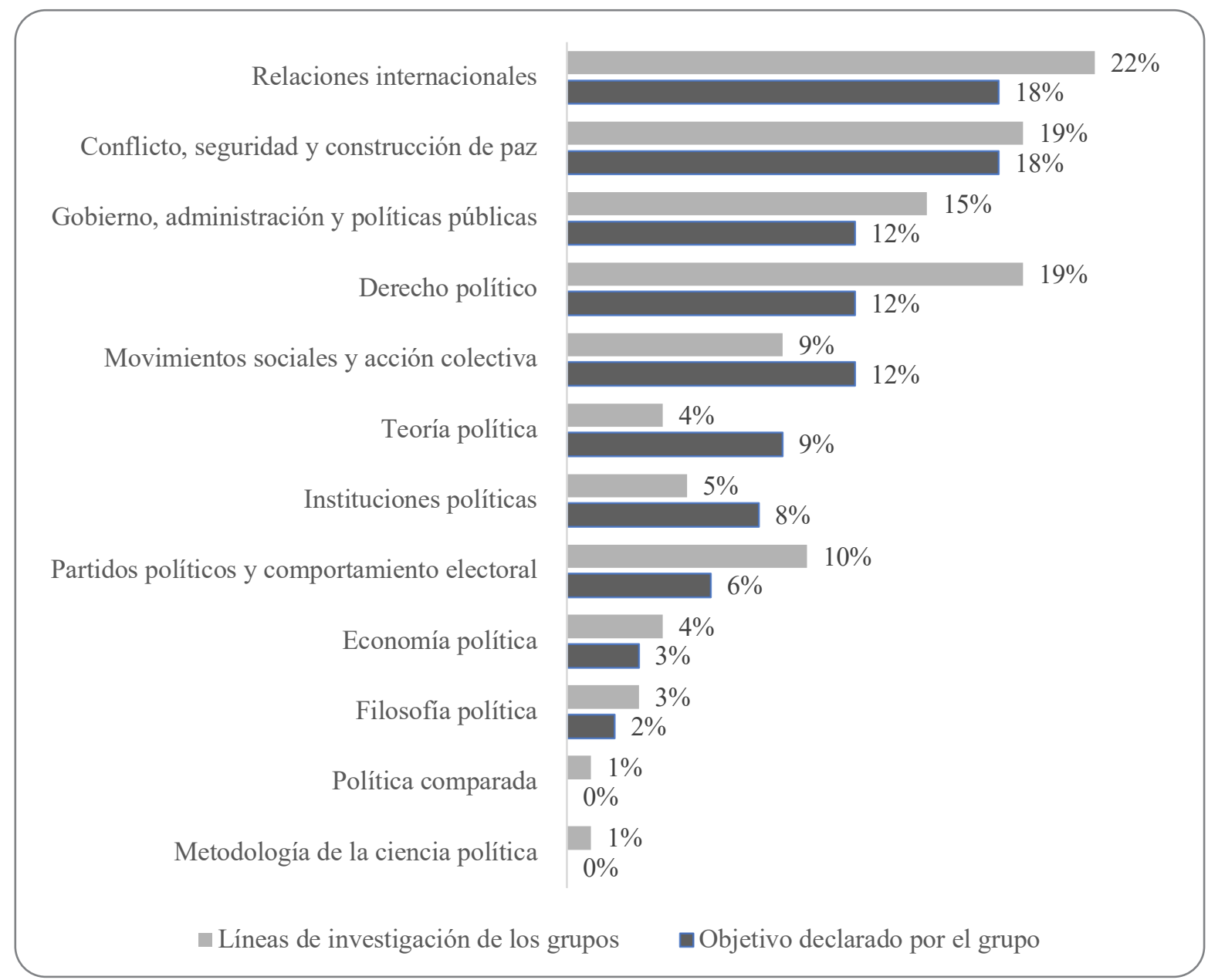

Fuente: elaboración propia con base en los resultados obtenidos por el Buscador Colciencias (2019).

La información de la figura 2 reafirma lo planteado anteriormente. Las agendas investigativas de los grupos de investigación giran o presentan un mayor interés en temas relacionados con las áreas de relaciones internacionales; conflicto, seguridad, construcción de paz y posconflicto; gobierno, administración y políticas públicas; y derecho político, movimientos sociales y acción colectiva. Estos datos permiten observar que en definitiva sí hay un desinterés en el país por los temas que engloban las áreas de metodología de la ciencia política y política comparada. Ello se puede deber precisamente a las falencias que se presentan en los currículos universitarios, en donde hay una desatención a la enseñanza de política comparada; lo cual indica, a su vez, una baja producción científica en temas relacionados a esta área (Leyva y Ramírez, 2015).

En sintonía con lo anterior, Geary et al. (2011) argumentan que América Latina se ha caracterizado por emprender tardamente estudios en lo concerniente a la política comparada. Los autores lograron exponer cómo se ha ido desarrollando esta importante área de la ciencia política y que, gracias a ello, hoy se cuenta con un selecto y reducido grupo de investigadores enfocados en la política comparada. A su vez, otro aspecto que debe llamar la atención es que en América Latina se encuentra solamente un posgrado a nivel de maestría en Política Comparada, otorgado por la Facultad Latinoamérica de Ciencias Sociales (FLACSO) sede Ecuador. 
Con lo discutido hasta el momento, se ha logrado responder la pregunta sobre cuáles son las principales áreas de estudio declaradas por los grupos de investigación en ciencia política en Colombia. Dicho esto, damos paso al siguiente apartado, que tiene como intención exponer la ubicación geográfica de los grupos de investigación. Asimismo, en la siguiente sección se presenta un panorama sobre el carácter público o privado de las universidades, en donde se registran y la relación de género inmersa en cada uno de los grupos de investigación.

\section{¿Dónde y quiénes hacen las investigaciones de ciencia política en Colombia?}

Con el panorama claro sobre las áreas en las cuales los grupos de investigación en Colombia desarrollan sus pesquisas, nos centramos en dar respuesta a la pregunta sobre dónde y quiénes investigan la ciencia política en el país. Para ello, en el presente apartado evidenciamos las zonas de la geografía colombiana en donde se encuentran inmersos los grupos de investigación avalados por Colciencias. A su vez, presentamos la relación entre universidades públicas y privadas desde donde se llevan a cabo las investigaciones de estos grupos. Por último, vemos la relación de género entre estos grupos, lo cual permite saber qué tan posicionados están los hombres y las mujeres en cuestión de liderazgo y pertenencia a grupos de investigación.

Para empezar, en lo que respecta a la ubicación geográfica de los grupos de investigación, encontramos algo muy similar a lo que expone Guzmán (2013):

La Ciencia Política en el país, como disciplina profesional, viene vislumbrando quizás el impacto de la política de descentralización implementada en el país a finales de la década de los años ochenta del siglo XX, la que posiblemente generó una demanda regional y local por los análisis de los procesos políticos, pero sin diálogo que la articulara. Respondió, sí, a iniciativas individuales y, en algunos casos, organizacionales, llenas de subjetividades y buenas intenciones personales e institucionales, más que a diálogos disciplinares que soportaran la apertura de los programas de Ciencia Política. (Guzmán, 2013, p. 140)

En este sentido, a partir de la observación de Guzmán (2013), el argumento para la apertura de programas de ciencia política también es válido y visible en el caso de los grupos de investigación. La figura 4 muestra una gran concentración de grupos de investigación en los principales departamentos políticos y económicos del país. Un 80 \% de los grupos de investigación se localizan en los departamentos de Cundinamarca, Antioquia y Valle del Cauca (ver figura 3), lo cual implica una investigación de ciencia política poco regionalizada.

Por consiguiente, se puede hablar de un centralismo académico por parte de los grupos de investigación, los cuales se ubican en las grandes ciudades de Colombia, especialmente en la capital del país: en Bogotá se concentran 37 grupos. Seguidamente, se encuentra Medellín (9 grupos), la segunda ciudad más poblada del país; y posteriormente, se encuentra la ciudad de Cali (7 grupos), la cual coincide en ser la tercera ciudad más poblada y con más grupos en ciencia política en Colombia.

De esta manera, se observa una distribución desigual en la ubicación de los grupos. En las regiones periféricas del país, la presencia de grupos de investigación es casi inexistente. Por tanto, se requiere que las universidades promuevan más la investigación y conformación de grupos que puedan ser reconocidos y avalados por Colciencias. En este aspecto vale la pena traer a colación el trabajo realizado por Macías (2017), cuyo análisis con relación a la investigación de la ciencia política en Colombia da cuenta del centralismo imperante a la hora de publicar libros y artículos, principalmente en Bogotá, Medellín y Cali. Este dato sugiere que hay una disparidad geográfica por parte de los y las investigadoras a la hora de no solo pertenecer a grupos de investigación, sino, también, de publicar y acceder a revistas indexadas en el país.

Por otro lado, más allá de la longitud e importancia territorial económica y política de las ciudades mencionadas, está el criterio de las políticas de investigación en las universidades. Estas son significativas a la hora de propiciar la creación tanto de semilleros de investigación internos de la universidad como grupos de investigación que estén registrados y sean reconocidos ante Colciencias. En tal sentido, es oportuno pensar en futuros trabajos e investigaciones que se concentren en analizar y comparar la promoción que están dando las universidades a la conformación de estos espacios académicos y de divulgación científica. 
Figura 3

Presencia y distribución geográfica de los grupos de investigación en ciencia política avalados por Colciencias

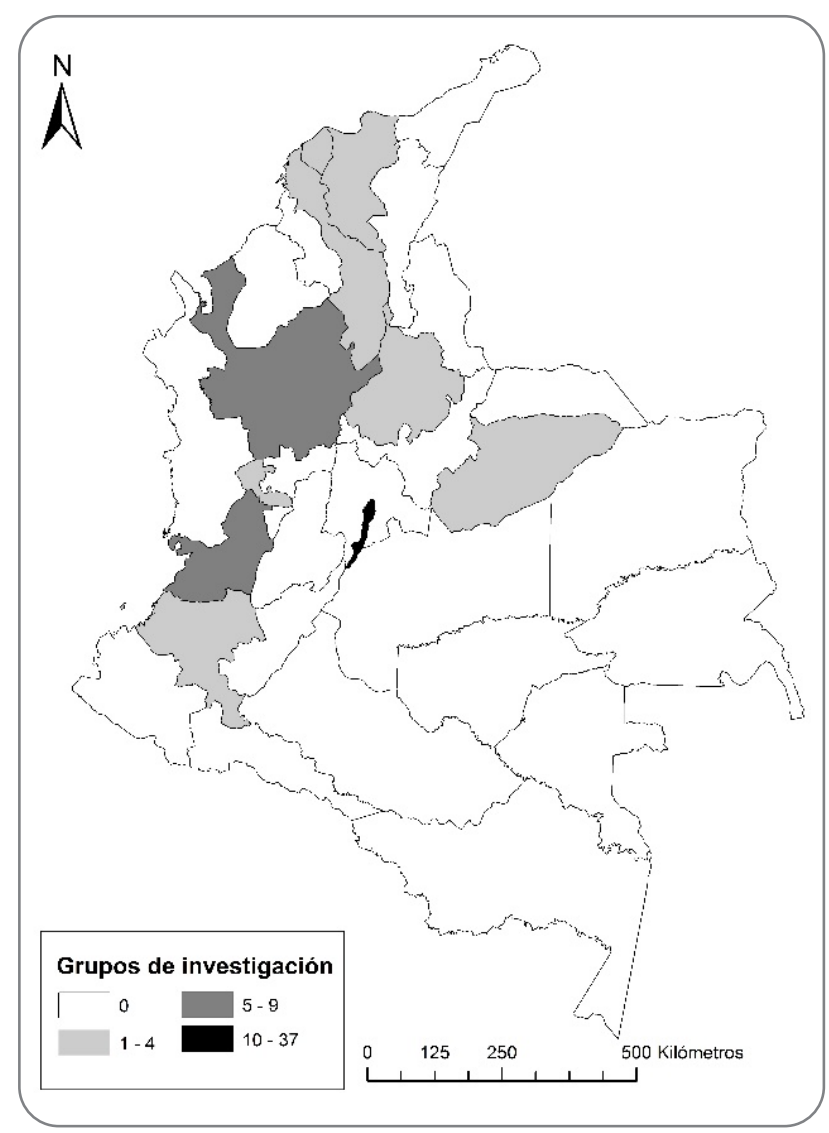

Fuente: elaboración propia con base en los resultados obtenidos por el Buscador Colciencias (2019).
En lo que respecta al carácter institucional de los grupos de investigación, se encontró que la relación no es muy dispareja entre las universidades públicas y privadas. La diferencia entre la tenencia de grupos de investigación en ciencia política en cada una es similar. Esta similitud se puede apreciar en la figura 4, de la cual se puede inferir que 32 grupos de investigación se localizan en universidades públicas y 34 en universidades privadas. Se alude con ello a una relación de reparto equitativo en el carácter institucional de la investigación de ciencia política en el país.

Con el anterior argumento se podría suponer que la investigación avalada por Colciencias no distingue el carácter de la universidad, sino -antes bien- los apoyos brindados por las universidades para fomentar la misma, así como de la planta docente de los programas. Estos elementos son importantes a la hora de asumir la responsabilidad de guiar un grupo de investigación y dar cuenta de los trabajos que se están realizando. En universidades públicas y privadas también podrían influir los perfiles académicos y formativos de los docentes de planta, el dominio en el uso de metodologías de investigación y el tiempo que ellos deben de asumir a tareas investigativas como asesoramiento de proyectos de grado o coordinación de grupos o semilleros de investigación.

\section{Figura 4}

Número de grupos según su carácter institucional y las ciudades en las cuales se ubican

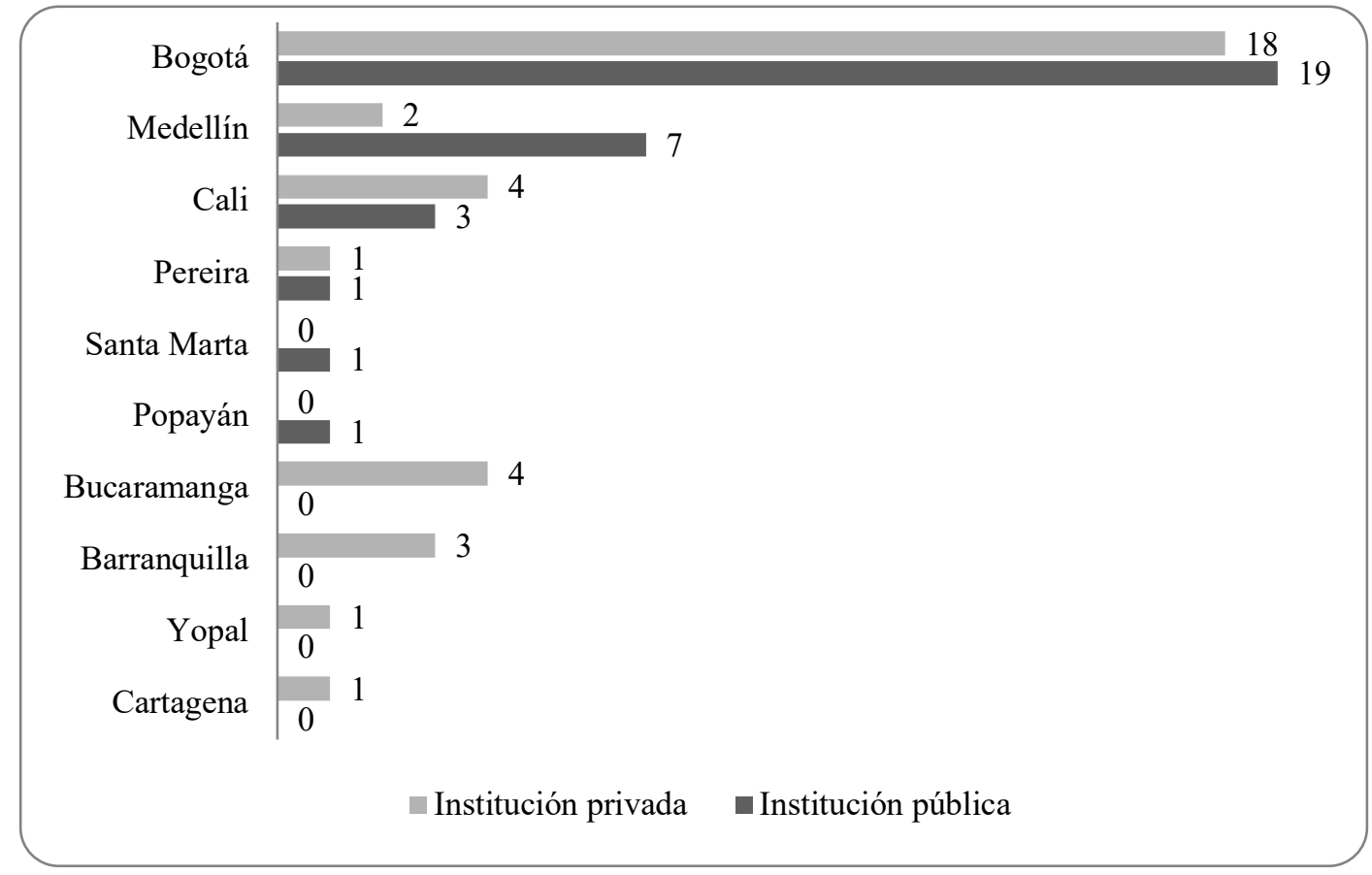

Fuente: elaboración propia con base en los resultados obtenidos por el Buscador Colciencias (2019). 
Por último, en cuanto a la relación de género inmersa en los grupos de investigación, se tomó en cuenta tanto a los líderes o coordinadores de grupo como a los integrantes. En la figura 5, se evidencia un porcentaje significativo en los miembros con género masculino en referencia al género femenino. El total de miembros hallados en los 66 grupos fueron de 1578 hombres y 1183 mujeres equivalente al $57 \%$ y $43 \%$ respectivamente.

En este aspecto solo podemos aludir a algunas investigaciones que se han desarrollado sobre las brechas de género en los trabajos de ciencia e investigación, los cuales señalan que la baja proporción de mujeres en la academia y en cargos directivos se debe a las tareas asignadas al género femenino referentes al hogar y el cuidado. Artículos promotores de la discusión se encuentran en Daza y Bustos (2008), quienes realizaron una exploración de los indicadores de género y ciencia en Colombia, así como las investigaciones de Tovar (2002, 2006, 2008) y Olaya (2003) referentes al acceso a la educación superior entre hombres y mujeres, la docencia y pertenencia a grupos de investigación en Colombia.

\section{Figura 5}

Relación de género de los líderes y miembros de los grupos de investigación en Ciencia Política

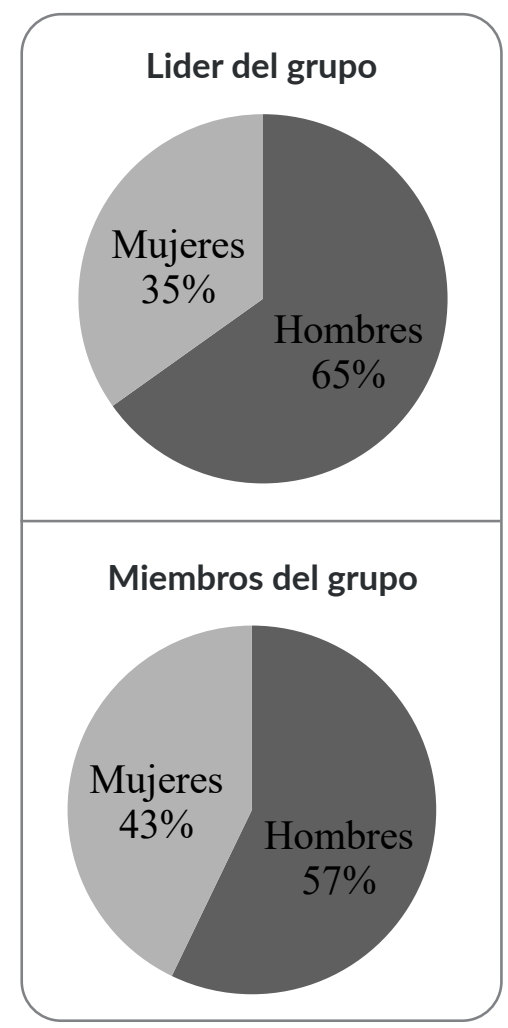

Fuente: elaboración propia con base en los resultados obtenidos por el Buscador Colciencias (2019).
Otros intentos por explicar la cuota de género en la ciencia política han sido realizados por autores como Ginther (2014), Gruberg y Sapiro (1979), Marijke y Kathryn (2007), Schuck (1969), entre otros, para el caso de Estados Unidos. Estos autores han reflexionado sobre la enseñanza de la ciencia política en el país anglosajón, agregando discusiones sobre la disparidad en el salario, la participación de la mujer en revistas y la formación académica entre hombres y mujeres. Sin embargo, dejamos abierta la discusión sobre el género en la investigación de la disciplina, pues creemos que es un tema que no se ha abordado lo suficiente y que merece plena atención.

\section{Conclusiones}

Con el estudio realizado, podemos señalar ciertas particularidades. En primer lugar, se observa que, luego de medio siglo del nacimiento, constitución, transformación y -porque no- una institucionalización de la ciencia política en Colombia, encontramos que existen procesos positivos para el fortalecimiento de la disciplina, uno de los cuales ha sido la instauración y reconocimiento de grupos de investigación. Con ello, se muestra la existencia de demarcadas líneas y áreas por las que transita la investigación de ciencia política en el país. Asimismo, se logró develar en qué regiones del país se están llevando a cabo los procesos investigativos de la disciplina avalados por Colciencias.

Por lo tanto, en esta investigación se presentó un primer avance sobre el estado de la investigación en Colombia sobre la ciencia política. En este sentido, el trabajo no está encaminado a ofrecer un conjunto de certezas, sino más bien a aportar algunas de las dudas e inquietudes que suscitan la reflexión constante sobre el estado de la ciencia política. En particular, sobre los temas que suscriben a la investigación al interior de los grupos de investigación en ciencia política en el país. Acorde con lo anterior, fue posible evidenciar que, a pesar de lo joven que es la ciencia política en el mundo de las ciencias sociales, se encuentra que esta disciplina ha logrado apropiar, consolidar y delimitar en su teoría y práctica un campo de estudio propio que se manifiesta en la construcción de las áreas presentadas en la tabla 1. Este es un producto de los diálogos entablados entre cultores de la disciplina y de los acuerdos a los que han llegado las asociaciones internacionales, regionales y nacionales en ciencia política. 
A pesar de ello, es importante señalar y aclarar que, así como en las demás ciencias sociales, en la ciencia política también el conocimiento está en continuo movimiento. Por lo tanto, no se puede considerar el saber producido en la disciplina como algo estático o concluido. Todo lo contrario, a medida que se desarrollan nuevos procesos sociales, políticos, culturales y económicos, se va abriendo espacio a nuevas indagaciones e investigaciones en el campo o áreas de la disciplina, las cuales obedecen precisamente a los contextos en los que los investigadores se desenvuelven ${ }^{8}$. En este sentido, se espera que las discusiones suscitadas hasta el momento en el ámbito de la disciplina se fortalezcan, nutran y perfeccionen cada día más.

Por otro lado, se reconoce que, si bien en el país hay trabajos escritos relacionados con las áreas de política comparada y metodología de la ciencia política, este ejercicio da cuenta de una necesidad que conlleva a hacer un llamado apremiante a investigar en estos temas. De esta manera, se nutrirá y propiciará un fortalecimiento hermenéutico sobre estos temas, pues -como se pudo observar en las figuras 1 y 2- estas son las áreas en las que se evidencia el grado más bajo de apropiación por los grupos de investigación. Por ende, como futura agenda investigativa, valdría la pena promover más investigaciones en Colombia con diferentes temas o áreas, haciendo uso del método comparado. Así, no solo se suplirá este vacío investigativo, sino que se rescatará la fortaleza que implica tener conocimiento de diferentes contextos y ponerlos a dialogar, revisando similitudes, diferencias, contradicciones, correlaciones, entre otros aspectos que se pueden adquirir por medio de la política comparada como eje característico de la ciencia política.

Otro aspecto que se logró observar es que, actualmente, la ciencia política colombiana se encuentra centralizada en cuanto a la investigación, lo cual muestra que, así como el país es centralizado, la ciencia política también lo es. En este sentido, como agenda investigativa, señalamos la urgencia de hacer investigaciones específicas que se concentren en revisar las diferentes políticas investigativas de las universidades para saber cuántos son los recursos que se asignan para el fomento de la investigación. También es importante ver cuáles son los incentivos que se brindan a la comunidad académica -tanto a docentes como estudiantes- que pertenece a espacios investigativos.
Por otro lado, para responder a la inquietud que surge sobre por qué en las principales ciudades del país es donde se encuentran más grupos de investigación registrados ante Colciencias, se podría plantear como agenda de investigación la opción de revisar la calidad del cuerpo docente de los pregrados de ciencia política en las diferentes ciudades: el grado máximo obtenido, si cuentan con más de un trabajo o si realizan funciones de asesoramiento en entidades públicas o privadas. Además de estos elementos, también revisar qué tan fuerte es la universidad en vinculación con temas de investigación, si es algo obligatorio o es algo opcional, entre otras circunstancias. Estos aspectos podrían ayudarnos a reconocer qué implica que en ciertas ciudades existan, o no, más grupos de investigación reconocidos, así como plantearnos si la cantidad de grupos de investigación o del establecimiento de políticas investigativas se puede ligar a lo que posiblemente se llame "cultura localizada de la investigación".

Por último, respecto al aspecto del género, se encontró que, si bien el porcentaje entre hombres y mujeres coordinadores de los grupos no posee una diferencia tan abismal, sí sería apropiado fortalecer las investigaciones en este tema. Más allá de que sea un tema de formación, pasión por la investigación o cualquier otro aspecto "racional", valdría la pena detenerse a observar las diferencias marcadas por el hecho de ser mujer, o ser hombre y las labores que históricamente han sido asignadas a cada uno. En este sentido, nos referimos específicamente a las labores de cuidado y del hogar asumidas, generalmente, por las mujeres, y por las cuales, es probable que se destine menos tiempo a labores investigativas. A manera de comentario final dejamos en consideración este acercamiento de lo que es el estado de la investigación en el país, para con ello empezar a entablar diálogos y consensos en cuanto a materia investigativa de la disciplina.

\section{Referencias}

Altman, D. (2005). La institucionalización de la ciencia política en Chile y América Latina: una mirada desde el sur. Revista de ciencia política (Santiago), 25(1), 3-15. http://doi.org/10.4067/So718090X2005000100001

Angel Baquero, S., Rico Noguera, J. C., y Caicedo Ortiz, J. A. (2019). Claves para problematizar el concepto de institucionalización en los estudios disciplinares: Una revisión crítica de la propuesta conceptual de 
David Altman. Civitas - Revista de Ciências Sociais, 19(3), 504-522. https://doi.org/10.15448/19847289.2019.3.33247

Barrientos del Monte, F. (2015) Crecimiento e institucionalización de la ciencia política en México. Revista de ciencia política (Santiago), 35(1), 95-120. http:// doi.org/10.4067/So718-090X2015000100006

Basabe-Serrano, S., y Huertas-Hernandéz, S. (2018). El estado de la investigación en ciencia política sobre América Latina. Revista Española de Ciencia Política, (47), 153-170. https://doi.org/10.21308/ recp. 47.06

Bejarano, A. M., y Wills, M. E. (2005). La ciencia política en Colombia: de vocación a disciplina. Revista de ciencia política (Santiago), 25(1), 111-123. http://doi. org/10.4067/So718-09oX2005000100008

Bentancur, N., y Mancebo, M. E. (2017). ¿Cómo se forma a los científicos políticos en Iberoamérica? Análisis de la oferta de titulaciones de grado en Ciencia Política en doce países. Revista española de ciencia política, (43), 161-185. https://doi.org/10.21308/ recp. 43.07

Buitrago Pinedo, L., Calle Oquendo, S., Romero Rúa, D., y Zapata Alzate, S. (2012). Aproximación cuantitativa del desarrollo de la Ciencia Política en Colombia, 1968-2012: un instrumento para el avance de la investigación [Trabajo de grado, Pregrado en Ciencia Política, Universidad de Antioquia]. Medellín.

Bulcourf, P., y Vázquez, J. C. (2004). La ciencia política como profesión. PostData: Revista de Reflexión y Análisis Político, 10, 255-304.

Caicedo Ortiz, J. A., Angel Baquero, S., y Cuellar Argote, J. A. (2015). Una ciencia política transdisciplinar en Colombia. Civilizar Ciencias Sociales y Humanas, 15(28), 135-150. https://doi. org/10.22518/16578953.284

Cuellar Argote, J. (2007). Un diagnóstico a la enseñanza de la ciencia política en Colombia, Civilizar Ciencias Sociales y Humanas, 7(13), 265-294. https:// doi.org/10.22518/16578953.780

Cuellar Argote, J., y Caicedo Ortiz, J. (2015). ¿Hacia dónde va la Ciencia Política? Reflexiones sobre la disciplina en Colombia. Universidad del Tolima.

Cuellar Argote, J., y Diaz Martinez, C. (2019). El uso del análisis automático de contenido desde Justin Grimmer y Brandon M Stewart: Colombia como parte de la producción académica internacional. Universidad del Tolima.

Daza, S., y Perez Bustos, T. (2008). Contando mujeres. Una reflexión sobre los indicadores de género y ciencia en Colombia. Antropología social, (10), 29-51.

Duque Daza, J. (2014). La ciencia política en Colombia. Análisis de los planes de estudio, sus áreas y sus énfasis. Papel Político, 19(2), 377-411. http://doi. org/10.11144/Javeriana.PAPO19-2.nppj

Duque Daza, J. (2015). La ciencia política en Colombia: la construcción de una comunidad académica (Primera ed.). Universidad del Valle.

Fortou, J. A., Leyva, S., Preciado, A. F., y Ramírez, M. F. (2013). Ciencia política en Colombia: Una revisión de la literatura sobre el Estado e historia de la disciplina en el país. En S. Leyva (Ed.), La Ciencia Política en Colombia: ¿iuna disciplina en institucionalización? (pp. 27-56). Universidad EAFIT

Geary, M., Bautista Lucca, J., y Pinillos, C. (2011). Tendencias en la producción del conocimiento sobre política comparada en América Latina. DelRevés.

Ginther, D. (2014). Gender differences in salary and promotion in Political Science. American Political Science Association. Unites States: APSA. http://citeseerx.ist.psu.edu/viewdoc/download?doi=10.1.1.595.4036\&rep=rep1\&type $=$ pdf

Goodin, R. E. (2010). The Oxford handbook of Political Science. Oxford University Press.

Goodin, R. E., y Klingemann, H. D. (1996). A new handbook of Political Science. Oxford University Press.

Greenstein, F., y Polsby, N. W. (1975). Handbook of Political Science. Addison-Wesley Pub.

Gruberg, M., y Sapiro, V. (1979). Participation by women in annual meetings, 1970-78. PS: Political Science \& Politics, 12(3), 318-324. doi:10.1017/ S1049096500007277

Guzmán, C. E. (2013). La ciencia política en el Caribe colombiano. En S. Leyva (Ed.), La Ciencia Política en Colombia: ¿una disciplina en institucionalización?, (pp. 139-160). Universidad Eafit.

Harto de Vera, F. (2006). Ciencia política y teoría política contemporáneas: una relación problemática. Editorial Trotta.

Laitin, D. (2004). The Political Science discipline. En E. Mansfield, y R. Sisson (Ed.), Democracy, autonomy and conflict in comparative and international politics (p. 11-40). Ohio State University Press.

Leiras, M., Abal Medina, J., y D'Alessandro, M. (2005). La ciencia política en Argentina: el camino de la institucionalización dentro y fuera de las aulas universitarias. Revista de ciencia política (Santiago), 25(1), 76-91. http://doi.org/10.4067/So718090X2005000100005

Leyva, S. (2013) La ciencia política en Colombia: ¿una disciplina en institucionalización? Universidad Eafit.

Leyva, S., y Ramírez, M. F. (2015). La ciencia política en colombia: Una disciplina en continua expansión. Revista de ciencia política (Santiago), 35(1), 71-94. http://doi.org/10.4067/So718090X2015000100005 
Losada, R. (2004). Reflexiones sobre el estado actual de la ciencia política en Colombia. Papel Político, 16(9), 9-27.

Manifiesto de Popayan. (2017). Anexo Documental. Anuario Latinoamericano. Ciencias Políticas y Relaciones Internacionales. (5), 231-233.

Marijke, B., y Kathryn, S. (2007). Gender and journal authorship in eight prestigious Political Science journals. Political Science \& Politics, 40(2), 347351. http://doi.org/10.1017/S1049096507070564

Nohlen, D. (2003). ¿Cómo enseñar ciencia política? Republicana. Política y Sociedad, (2), 3-7.

Olaya, D. (2003). La creciente participación de la mujer en la investigación en ciencia y tecnología a finales de la última década. Colombia, Ciencia y Tecnología, 21(1), 16-25. Colciencias.

Organización de las Naciones Unidas para la Educación, la Ciencia y la Cultura - Unesco (1950). Contemporary Political Science: A Survey of Methods, Research and Teaching. UNESCO.

Prelot, M. (1964), La ciencia política. EUDEBA.

Ravecca, P. (2019). The politics of political science: Re-writing Latin American experiences. Routledge.

Schuck, V. (1969). Women in Political Science: some preliminary observations. Political Science \& Politics, 2(4), 642-653. http://doi.org/10.1017/ S1049096500010337

Tovar, P. (2002). Género y ciencia en Colombia: algunos indicadores. En Colciencias, Colombia Ciencia y Tecnología, 20(2), 3-10. Colciencias.

Tovar, P. (2006). Indicadores nacionales de género, ciencia y tecnología. [Memorias] Seminario: Las mujeres colombianas en el sistema de ciencia y tecnología: obstáculos y logros. Instituto Colombiano de Antropología e Historia

Tovar, P. (2008). La mujer colombiana en la ciencia y la tecnología. ¿Se está cerrando la brecha? $A R B O R$ Ciencia, Pensamiento y Cultura, 184(733), 835844. https://doi.org/10.3989/arbor.2008.i733.228

Vallès, J. (2006). Ciencia política: una introducción. Ariel.

Wallerstein, I. (1996). Abrir las ciencias sociales. Informe de la Comisión Gulbenkian para la reestructuración de las ciencias sociales. Siglo XXI editores y UNAM.

\section{Notas}

1 Antes del año en mención, se puede hablar de una ciencia política incipiente, pero con grandes reflexiones teóricas en manos de los pensadores clásicos, tales como Platón, Maquiavelo, Rousseau, Hobbes, Locke, entre otros. Los cuales -al menos en el tema de la política- centraban sus disertaciones en lo re- ferente al Estado, su conformación y la garantía de la seguridad como principal objetivo (Vallès, 2006).

2 Al respecto, Wallerstein (1996) señala que la institucionalización de las disciplinas viene acompañada tanto del proceso de enseñanza como del proceso investigativo. Este último se refiere a la creación de publicaciones especializadas, la construcción de asociaciones de estudiosos, la creación de colecciones y bibliotecas. Esta idea es retomada para la ciencia política por Altman (2005); Fortou et al. (2013), entre otros.

3 Es así que planteamos un enfoque de análisis que no se ha explorado con profundidad en otros dossiers como el volumen 5 del Anuario LatinoamericanoCiencias Políticas y Relaciones Internacionales (2017), o las entregas especiales del 2005 y 2015 de la Revista de Ciencia Política vol. 25, no. 1 y vol. 35, no. 1 respectivamente.

4 Colciencias es el Departamento administrativo de ciencia, tecnología e innovación de Colombia. Encargado de promover políticas públicas en los temas de ciencia, tecnología e innovación, y llevar el registro de los investigadores y producción intelectual de los centros de enseñanza o universidades.

5 Cada una de las áreas propuestas por los diversos autores presentados en los tres momentos se encuentran referenciadas en la tabla 1.

6 La lista completa de los comités de investigación de IPSA se encuentra en: https://www.ipsa.org/page/ research-committees-rc-complete-list-researchcommittees

7 Para ampliar la información de los grupos de investigación de ALACIP puede ingresar a: http://alacip. org/?page_id=2256\#grupo

8 Cuando se habla de contextos se hace referencia al entorno histórico, político, económico y social en el que un investigador realiza sus pesquisas académicas; por lo tanto, se debe reconocer que cada saber expresado en forma de nuevo conocimiento obedece a un contexto determinado. 